\title{
Etiología de episodios de neutropenia febril en pacientes adultos con cáncer hematológico y de órganos sólidos en el Hospital Clínico Universidad Católica, Santiago-Chile
}

\author{
Ricardo Rabagliati B., Gino Fuentes L., Eric Orellana U., Jorge Oporto C., Isabel Domínguez M., \\ Rosana Benítez G., Igor Aedo C., Germán Ramos G., Marcelo Garrido S. y Patricia García C.
}

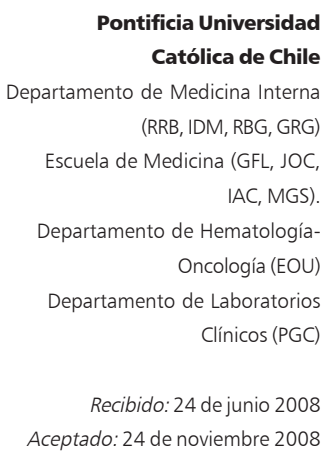

Correspondencia a: Ricardo Rabagliati Borie rabagli@med.puc.cl

Etiology of febrile neutropenia episodes among cancer patients from Hospital Clinico Universidad Catolica, Santiago-Chile

Introduction: The surveillance of febrile neutropenia $(\mathrm{FN})$ episodes in every center allows adapt the antibiotic therapy guidelines to local epidemiology. Aim: To characterize clinical features and compare the FN etiology between hematological cancer (HC) and solid organ cancer (SOC) in our center. Patients and Methods: Surveillance study in adult patients with FN admitted to Hospital Clinico Universidad Católica, in Santiago, Chile, from January 2004 to August 2007. Results: 154 FN episodes corresponding to 87 patients were included. Mean age: $47 \pm 6$ years-old; $71 \%$ had HC and 29\% SOC. A clinical and/or microbiologically documented infection was recognized in $76 \%$. Gastrointestinal $31.5 \%$, upper respiratory $30.3 \%$ and lower respiratory $16.9 \%$ were the more frequent clinical focus. In $30.5 \%$ blood culture resulted positive: gram negative rods $51 \%$, gram positive cocci $41 \%$ and yeasts $8 \%$; being Escherichia coli $22 \%$, S. coagulase negative $(\mathrm{SCoN}) 20 \%$ and Klebsiella pneumoniae 12\% most frequent bacteria; 22.2\% Enterobacteriaceae were ESBL producers and 55.6\% SCoN were methicillin resistant. In $18.3 \%$ of FN episodes the etiology was not established. Highest mortality was observed in episodes with microbiologically documented infection $(14.5 \%$ vs $1.3 \%, \mathrm{p}<0.005)$. A clinical observed focus and positive blood cultures were more frequently obtained among HC than SOC associated episodes: $37.3 \%$ vs $13.6 \% ;(\mathrm{p}<0.01)$ and $67.2 \%$ vs 50\%; $(\mathrm{p}=0.045)$, respectively. Conclusions: The etiological profile of FN in our center and the necessity to continue the surveillance was described. Future studies are needed regarding risk factors of invasive infection that have worst prognosis.

Key words: Neutropenia, etiology, cancer, chemotherapy, infections.

Palabras clave: Neutropenia, etiología, cáncer, quimioterapia, infecciones.

\section{Introducción}

$\mathrm{L}$ a neutropenia febril (NF) es una complicación frecuente de los pacientes que reciben quimioterapia por cáncer, se observa hasta en $40 \%$ de ellos $^{1}$, dependiendo de varios factores tales como: edad, tipo de cáncer, fármacos empleados en la quimioterapia, co-morbilidades, etc ${ }^{2}$. Para los pacientes significa aumentar y prolongar hospitalizaciones, elevar el costo del tratamiento, retrasar los nuevos ciclos de quimioterapia e incluso mortalidad, la que sobrepasa el $30 \%$ en aquellos que presentan bacteriemia ${ }^{3}$.

En los pacientes con NF se documentan infecciones por bacterias, hongos y menos frecuentemente por virus. Las bacterias son los agentes etiológicos más frecuentemente involucrados en las fases iniciales de la NF, aislándose tanto bacilos gramnegativos
(BGN) como cocáceas grampositivas (CGP) $)^{4}$ En cambio, las infecciones por hongos son infrecuentes como infección primaria, salvo Candida sp, que ocasionalmente puede ser identificada en hemocultivos ${ }^{3}$. El predominio de BGN o CGP como agentes más frecuentes de bacteriemia ha sido variable a lo largo del tiempos: predominio de BGN durante los años 70 , mientras que durante los años 80 se reportó una emergencia progresiva de infecciones por CGP hasta llegar a ser el grupo predominante durante la década de los $90^{6}$. Sin embargo, durante los últimos años algunos centros han destacado el rol de las infecciones polimicrobianas en las que participan predominantemente $\mathrm{BGN}^{7}$. En Chile hay pocos datos de vigilancia de infecciones en pacientes neutropénicos adultos; en la casuística publicada del Hospital Del Salvador, en Santiago, en pacientes con leucemia predominan los BGN como grupo 
de microorganismos más frecuentes en dos períodos de evaluación entre los años 1990 y $2000^{8}$.

Recomendaciones nacionales e internacionales sugieren diferentes esquemas antimicrobianos según la condición del paciente con $\mathrm{NF}^{4,9}$, pero para establecer su aplicabilidad se recomienda conocer la etiología local de los episodios de NF en cada centro9; los esquemas de enfrentamiento empírico deben ser adaptados a la necesidad de los pacientes de cada institución bajo la premisa de que la epidemiología podría ser diferente en los distintos hospitales.

Desde esta perspectiva, el objetivo del presente trabajo fue identificar la etiología de las infecciones en los episodios de NF en pacientes adultos del Hospital Clínico de la Universidad Católica (HCUC) que permitiera establecer de mejor forma las guías de tratamiento empírico de estos pacientes. Como objetivos secundarios se planteó comparar la etiología de los episodios de NF entre pacientes con cáncer hematológico $(\mathrm{CH})$ vs cáncer de órganos sólidos (COS) y evaluar la tendencia, en el tiempo, de las bacteriemias en NF al comparar con datos de estudio previos.

\section{Pacientes y Métodos}

El HCUC es un recinto hospitalario de nivel terciario de atención, ubicado en la ciudad de Santiago, Chile, donde anualmente se hospitalizan alrededor de 25.000 pacientes; cuenta con 493 camas de las cuales 58 corresponden preferentemente a cuidados de pacientes oncológicos adultos. Junto al HCUC existe un centro ambulatorio de diagnóstico y terapia del cáncer "Nuestra Señora de la Esperanza" al que anualmente consultan alrededor de 9.000 pacientes para evaluación o terapia oncológica, administrándose por año 1.800 ciclos de quimioterapia ambulatorios y 1.300 ciclos hospitalizados en HCUC donde también son referidos para procedimientos diagnósticos o terapéuticos, incluyendo manejo de NF.

Se diseñó un estudio prospectivo de vigilancia de los episodios de NF en pacientes en tratamiento de quimioterapia por $\mathrm{CH}$ o COS hospitalizados en el HCUC, entre enero 2004 y agosto 2007. Se incluyeron pacientes adultos ( $\geq 15$ años) cursando NF definida como temperatura mayor o igual a $38^{\circ} \mathrm{C}$ y un recuento absoluto de neutrófilos (RAN) menor o igual a 500 células $/ \mathrm{mm}^{3}$.

Se confeccionó una ficha para registro de datos de cada paciente, en la que se consignaron datos biodemográficos de edad y sexo, antecedentes oncológicos como tipo de quimioterapia, patología oncológica, presencia de mucositis según clasificación $\mathrm{OMS}^{10}$, RAN; resultados de la evaluación clínico-microbiológica realizada de acuerdo a las pautas habituales de manejo de NF en HCUC, considerando los hallazgos de los primeros cinco días del diagnóstico de NF: foco clínico de infección, hallazgos de estudios imagenológicos, resultados de hemocultivos, urocultivo y otros, según les fuese solicitado a criterio de su médico tratante (cultivo de secreción, estudio de virus respiratorios, serologías, etc), informe de susceptibilidad in vitro en aquellos casos con cultivos positivos, tratamientos antimicrobianos recibidos y condición de egreso.

Los episodios de NF fueron catalogados como "infección clínicamente documentada" si se evidenciaban elementos clínicos y/o estudio de imágenes con focalización; "infección microbiológicamente documentada" si se demostraba microorganismo a través de cultivos u otro método diagnóstico y "fiebre de origen desconocido" (FOD) en caso que ni las manifestaciones clínicas, ni el estudio de imágenes ni microbiológico permitiera explicar el origen de la fiebre.

Frente a la identificación de Staphylococcus coagulasa negativa $(S C o N)$, a modo de no incluir hemocultivos contaminados, se aplicaron los siguientes criterios de exclusión: cultivos positivos después de 27 horas de incubación, un cultivo positivo con otro concomitante negativo o dos especies diferentes en dos hemocultivos positivos simultáneos ${ }^{11}$. En caso que se hubiese tomado sólo una muestra de hemocultivo con resultado positivo a $S \mathrm{CoN}$ se consideraba la opinión del médico tratante según si decidía incluir su cobertura en el esquema antimicrobiano.

Para efecto de evaluar tendencias, se rescataron datos previamente comunicados por nuestro grupo ${ }^{12}$ de un estudio retrospectivo de bacteriemias en pacientes con NF del 2001 al 2003 en el HCUC donde se aplicaron los mismos criterios de inclusión y exclusión que en el presente estudio.

Análisis estadístico: se construyó una base de datos con el programa Access 2003 y se utilizó el programa SPSS 13.0 para realizar el análisis estadístico. Los resultados de las variables nominales se expresan en porcentajes y de las numéricas en promedio \pm desviación estándar (DS) e intervalo. En el análisis comparativo de los pacientes con $\mathrm{CH}$ versus los con $\mathrm{COS}$ se utilizó el test de t-Student para las variables continuas y para las variables discontinuas test de $\chi^{2}$ o test de Fischer. Se consideró como diferencia estadísticamente significativa el valor de $\mathrm{p}$ menor o igual a 0,05 .

\section{Resultados}

Se incluyeron 154 episodios de NF correspondientes a 87 pacientes. Las características generales de los pacientes incluidos se muestran en la Tabla 1. La mayor parte correspondió a pacientes con $\mathrm{CH}$, siendo 
Tabla 1. Características generales de 154 pacientes con episodios de NF

\begin{tabular}{|c|c|c|}
\hline & $\mathbf{n}$ & (\%) \\
\hline Edad, años [promedio \pm DS (intervalo)] & $47,6 \pm 14,8$ & $(17-82)$ \\
\hline Sexo femenino & 78 & $(50,6)$ \\
\hline Cáncer hematológico & 110 & $(71,4)$ \\
\hline Leucemia mieloide aguda & 66 & $(60,0)$ \\
\hline Linfoma no Hodgkin & 19 & $(17,3)$ \\
\hline Leucemia linfática aguda & 12 & $(10,9)$ \\
\hline Linfoma de Hodgkin & 11 & $(10,0)$ \\
\hline Otro & 2 & $(1,8)$ \\
\hline Cáncer de órganos sólidos & 44 & $(28,6)$ \\
\hline Mama & 11 & $(25,0)$ \\
\hline Pulmón & 11 & $(25,0)$ \\
\hline Testículo & 6 & $(13,6)$ \\
\hline Colon & 4 & $(9,1)$ \\
\hline Estómago & 3 & $(6,8)$ \\
\hline Endometrio & 3 & $(6,8)$ \\
\hline Otro & 6 & $(13,6)$ \\
\hline Mucositis & 57 & $(37,0)$ \\
\hline Grado I & 36 & $(63,2)$ \\
\hline " II & 15 & $(26,3)$ \\
\hline " III & 4 & $(7,0)$ \\
\hline "IV & 2 & $(3,5)$ \\
\hline Portación CVC & 142 & $(92,2)$ \\
\hline RAN céls $/ \mathrm{mm}^{3}$ [promedio \pm DS (intervalo)] & $194 \pm 165(0$ & $-500)$ \\
\hline RAN $\leq 100$ céls $/ \mathrm{mm}^{3}$ & 56 & $(36,4)$ \\
\hline
\end{tabular}

\begin{tabular}{|c|c|c|}
\hline $\begin{array}{l}\text { Infección clínicamente documentada } \\
\text { Foco clínico }\end{array}$ & $\mathbf{n}$ & $(\%)$ \\
\hline Gastrointestinal & 28 & $(31,5)$ \\
\hline Respiratorio alto & 27 & $(30,3)$ \\
\hline Respiratorio bajo & 15 & $(16,9)$ \\
\hline Piel y tejidos blandos & 15 & $(16,9)$ \\
\hline Oral & 2 & $(2,2)$ \\
\hline Urinario & 1 & $(1,1)$ \\
\hline Otro & 1 & $(1,1)$ \\
\hline
\end{tabular}

leucemia mieloide aguda la enfermedad más frecuente en este grupo y cáncer de mama y pulmón los más frecuentes entre los COS. Sobre 90\% de los episodios se presentaron en pacientes con catéter venoso central (CVC). En 37\% de los episodios se observó mucositis, siendo más frecuentes las catalogadas leves a moderadas (grado I y II) sobre las graves (grado
III y IV). En 36\% de los casos el RAN fue menor o igual a 100 céls $/ \mathrm{mm}^{3}$.

En 117 de los episodios (76\%) se identificó una infección como etiología de la NF: en 41 episodios $(26,7 \%)$ por la presencia de al menos un foco clínico como único hallazgo, en $28(18,2 \%)$ por la identificación de al menos un microorganismo y en 48 (31,1\%) por la identificación simultánea de foco clínico más documentación microbiológica de infección. Los restantes 37 casos (24\%) fueron catalogados como FOD.

Focalización clínica. En los 89 episodios $(57,8 \%)$ en que se documentó clínicamente una infección, los focos evidenciados fueron, en frecuencia decreciente: gastrointestinal, respiratorio alto (faringitis, rinitis, sinusitis), respiratorio bajo (bronquitis, neumonía); piel y tejidos blandos (celulitis, infección de sitio de inserción de CVC y flebitis) y oral, urinario u otro (Tabla 2). En 7,8\% de los episodios se identificó más de un foco clínico en forma simultánea, predominando la concomitancia de uno gastrointestinal con otro, ya fuera respiratorio bajo (n: 3$)$, piel (n: 2) y respiratorio alto (n: 1).

Hallazgos microbiológicos. En un total de 154 episodios de NF se identificaron hemocultivos positivos en $47(30,5 \%)$, incluyendo un episodio en que se aislaron dos agentes simultáneamente. En 24 episodios se obtuvo otra muestra clínica que resultó con cultivo positivo, ya sea en forma adicional al hemocultivo o como documentación microbiológica única: orina $48 \%$, secreción respiratoria 32\% y secreción de lesión cutánea $10 \%$. Los aislados en hemocultivos correspondieron a BGN (51\%), CGP (41\%) y levaduras (8\%), siendo los agentes predominantes: Escherichia coli, SCoN, Klebsiella pneumoniae y Pseudomonas aeruginosa (Tabla 3). De acuerdo a los criterios señalados se excluyeron tres hemocultivos positivos a $S C o N$ : en dos casos por haber obtenido hemocultivo periférico negativo y central positivo y en otro caso por cultivo periférico positivo a las $44 \mathrm{hr}$ con cultivo central negativo. Adicionalmente, en 13 episodios se identificó un agente viral: parainfluenza (n: 5), herpes simplex (n: 4), virus respiratorio sincicial (n: 3 ) e influenza-A (n: 1). En los casos con infección demostrada por virus respiratorios el cuadro clínico era compatible con un foco respiratorio alto. En el caso del paciente con influenza A, clínicamente presentaba focos respiratorio alto y gastrointestinal y se obtuvo hemocultivo positivo a Candida albicans. En ninguno de los otros episodios con virus respiratorio se obtuvo hemocultivos positivos. En los casos con herpes simplex las lesiones muco-cutáneas eran de extensión limitada al área perioral por lo que probablemente no eran la causa de la fiebre. 
Susceptibilidad antimicrobiana. Cuatro de 18 $(22,2 \%)$ enterobacterias detectadas en sangre eran resistentes a cefalosporinas de $3^{\text {a }}$ generación, correspondientes a E. coli (n: 3) y Enterobacter cloacae (n: 1), todas productoras de $\beta$-lactamasa de espectro expandido (BLEE). No hubo aislamiento de otros BGN multiresistentes. Respecto a CGP, 5 de $9 S \mathrm{CoN}$ resultaron resistentes a oxacilina $(55,6 \%)$ y uno de dos E. faecium identificados era resistente a vancomicina (ERV) con patrón van-B.

En la búsqueda dirigida de posibles factores asociados a bacteriemia por microorganismos resistentes se identificó que los cuatro pacientes con aislamiento de BGN BLEE positivas habían tenido alguna hospitalización previa en las últimas cuatro semanas por diferentes motivos: episodios de NF en dos casos, infección de sitio de inserción de CVC en un caso y en otro paciente para administración de quimioterapia. En cambio, en los 14 episodios con hemocultivos positivos a enterobacterias no BLEE positivas, habían menos casos con hospitalizaciones previas, pero sin alcanzar diferencia estadísticamente significativa (100 vs 79\%; $\mathrm{p}=0,55)$. En el caso del paciente con ERV sólo se identificó una hospitalización en el HCUC nueve meses antes.

Letalidad y factores asociados. Se observó mayor mortalidad en aquellos episodios en que se obtenía documentación microbiológica de infección versus aquellos en que no se obtenía $(14,5$ vs $1,3 \%$, $\mathrm{p}<0,005)$. $\mathrm{Si}$ bien se observó una mayor letalidad cuando se identificó clínicamente una infección comparado con episodios en que no se precisó foco, esta diferencia no alcanzó a ser estadísticamente significativa $(10,1$ vs $4,6 \% ; \mathrm{p}=0,24)$. No falleció paciente alguno cursando una NF catalogada como FOD.

Al comparar episodios de NF en pacientes con COS vs $\mathrm{CH}$ (Tabla 4), eran de edades similares, había mayor frecuencia de sexo femenino en el grupo de COS, mayor profundidad de neutropenia y mucositis en los episodios de NF de pacientes con CH. También fue más frecuente documentar infecciones tanto clínica como microbiológicamente en el grupo de $\mathrm{CH}$ versus COS, resultando significativamente más frecuente la identificación de foco clínico, infección en piel y tejidos blandos, la obtención de hemocultivos positivos y la documentación clínica y microbiológica simultánea. En ambos grupos fue más frecuente la bacteriemia por BGN sobre CGP, con una tendencia a observarse mayor frecuencia de bacteriemia por CGP en los pacientes con $\mathrm{CH}$. Cabe destacar que los casos de candidemia se presentaron solamente en el grupo de $\mathrm{CH}$.

Al comparar las bacteriemias en el período en estu-
Tabla 3. Frecuencias de 49 microorganismos identificados en hemocultivos positivos correspondientes a 47 casos de 154 episodios de NF

\begin{tabular}{|lrr|}
\hline Agente hemocultivo & n & (\%) \\
\hline Escherichia coli & 11 & $(22,4)$ \\
\hline Staphylococcus coagulasa negativa & 10 & $(20,4)$ \\
\hline Klebsiella pneumoniae & 6 & $(12,2)$ \\
\hline Pseudomonas aeruginosa & 4 & $(8,2)$ \\
\hline Streptococcus grupo viridans & 4 & $(8,2)$ \\
\hline Burkholderia cepacia & 2 & $(4)$ \\
\hline Staphylococcus aureus & 2 & $(4)$ \\
\hline Candida albicans & 2 & $(4)$ \\
\hline Enterobacter sp & 1 & $(2)$ \\
\hline Enterococcus faecium & 1 & $(2)$ \\
\hline Enterococcus fecalis & 1 & $(2)$ \\
\hline Streptococcus dysgalactiae sp (grupo G) & 1 & $(2)$ \\
\hline Escherichia fergusonii & 1 & $(2)$ \\
\hline Actinomyces odontolyticus & 1 & $(2)$ \\
\hline Candida kefyr & 1 & $(2)$ \\
\hline Candida krusei & 1 & $(2)$ \\
\hline
\end{tabular}

Tabla 4. Comparación de características generales y etiología de episodios de NF en pacientes con cáncer de órganos sólidos (COS) versus cáncer hematológico (CH)

\begin{tabular}{|c|c|c|c|}
\hline & $\begin{array}{c}\text { COS } \\
n=44 \\
n \quad(\%)\end{array}$ & $\begin{array}{c}\text { CH } \\
n=110 \\
n \quad(\%)\end{array}$ & $\mathbf{p}$ \\
\hline Edad, años [promedio \pm DS] & $50,1 \pm 14,9$ & $46,7 \pm 14,6$ & 0,9 \\
\hline Sexo femenino & $29(65,9)$ & $47(42,7)$ & 0,01 * \\
\hline RAN $($ céls/mm³) [promedio \pm DS] & $208 \pm 168$ & $188 \pm 164$ & 0,5 \\
\hline RAN $<100$ céls $/ \mathrm{mm}^{3}$ & $13(29,5)$ & $43(39,0)$ & 0,3 \\
\hline Mucositis & $6(13,6)$ & $30(27,3)$ & 0,07 \\
\hline Infección clínicamente documentada & $22 \quad(50)$ & $74(67,2)$ & $0,045^{*}$ \\
\hline Gastrointestinal & $8(18,1)$ & $20(18,2)$ & 1 \\
\hline Respiratoria alta & $7(15,9)$ & $20(18,2)$ & 0,8 \\
\hline Respiratoria baja & $4 \quad(9,1)$ & $14(12,7)$ & 0,6 \\
\hline Piel y tejidos blandos & $1 \quad(2,3)$ & $17(15,4)$ & $0,02 *$ \\
\hline Oral & 0 & $3(2,7)$ & 0,6 \\
\hline Urinaria & $1 \quad(2,3)$ & 0 & 0,3 \\
\hline Otra & $1(2,3)$ & 0 & 0,3 \\
\hline Infección microbiológicamente documentada & $18(40,9)$ & $58(52,7)$ & 0,2 \\
\hline Hemocultivo positivo & $6(13,6)$ & $41(37,3)$ & 0,004 * \\
\hline BGN & $4 \quad(9,1)$ & $21(19,1)$ & 0,15 \\
\hline CGP & $2(4,5)$ & $18(16,4)$ & 0,06 \\
\hline Candida sp & 0 & $4(3,6)$ & 0,3 \\
\hline Infección clínica y microbiológicamente documentada & $8(18,2)$ & $40(36,4)$ & $0,03^{*}$ \\
\hline Fiebre de origen desconocido & $12(27,2)$ & $25(22,7)$ & 0,5 \\
\hline Fallece & $2(4,5)$ & $10 \quad(9,1)$ & 0,5 \\
\hline
\end{tabular}




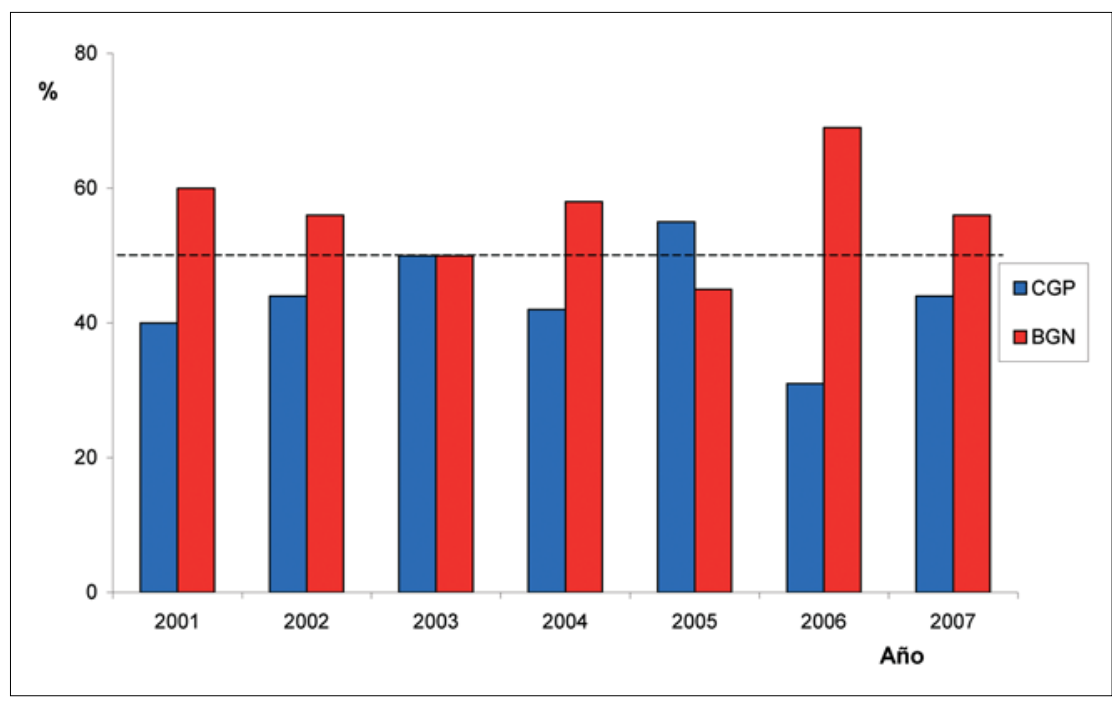

Figura 1. Porcentaje de identificación de hemocultivos positivos con CGP y BGN por año. La línea intermitente marca el 50\% a modo de destacar el predominio de BGN con excepción de los años 2003 y 2005. dio 2004-2007 con datos históricos a partir del 2001², se observó predominio de BGN con excepción de una situación de equilibrio durante 2003 y predominio de CGP el año 2005 pero, en general, con la tendencia a predominar la identificación de BGN sobre CGP con un promedio de $56 \%$ sobre $44 \%$ (Figura 1 ).

\section{Discusión}

A través de esta serie se logró caracterizar el perfil etiológico de los episodios de NF de pacientes adultos post-quimioterapia de $\mathrm{CH}$ y $\mathrm{COS}$ atendidos en nuestro centro, con una alta frecuencia de documentación de infección ya fuera clínica o microbiológicamente, constatándose mayor gravedad en pacientes con $\mathrm{CH}$ sobre aquellos con COS.

En más de $50 \%$ de los episodios se identificó un foco clínico que podría explicar la fiebre. En datos publicados en la literatura médica se identifica un foco clínico con enorme variabilidad, desde 13,2\% hasta $70 \%{ }^{8,13,14,16}$. Estas diferencias pueden estar dadas por el momento en que se realiza la evaluación y los criterios para documentar clínicamente una infección. En muchas oportunidades no se puede asegurar con certeza que algunos síntomas de los pacientes correspondan a un foco clínico de infección o sean síntomas originados por mucositis que puede confundir con foco oral, gastrointestinal y/o respiratorio alto. La evaluación inicial de pacientes con NF puede ser errónea hasta en $33 \%$ de los $\operatorname{casos}^{15}$. Por lo tanto, es pilar fundamental realizar una anamnesis y examen físico detallados en cada episodio y repetido en el curso de los días, teniendo en cuenta las dificultades que presentan estos pacientes en expresar focos de infección. Nos parece importante insistir en la búsqueda y reconocimiento de un foco clínico a fin de adaptar el esquema antimicrobiano con cobertura específica para el foco identificado 9 .

La documentación microbiológica, en particular el aislado de microorganismos a partir de sangre, permite el diagnóstico certero de una infección como causa de fiebre. En esta serie, en $30,5 \%$ de los episodios se obtuvo hemocultivos positivos, frecuencia similar a la publicada en el exterior: 22 a $35 \%{ }^{7,15,17}$. En nuestra serie destaca el predominio de BGN sobre CGP, coincidiendo con otros datos chilenos ${ }^{8}$, pero resulta diferente a la tendencia de predominio de CGP señalada en la literatura internacional desde la década de los $90^{3,6}$. Como explicaciones al predominio de CGP se ha planteado el mayor uso de catéteres intravasculares de larga duración, quimioterapias más enérgicas con mayor incidencia de mucositis de alto grado y el uso de quimioprofilaxis con quinolonas ${ }^{18,19}$. No resulta claro cuál sería la razón para este predominio de BGN sobre CGP en nuestro centro; se podría teorizar que la ausencia de uso rutinario de profilaxis con quinolonas tendría algún impacto.

Staphylococcus coagulasa negativa resultó el agente más frecuentemente detectado en sangre, coincidiendo con lo descrito en la literatura médica ${ }^{18}$. La interpretación del aislado de SCoN desde hemocultivos en pacientes con NF es polémica dado que, con alta frecuencia no corresponde más que a una contaminación ${ }^{20}$. El uso de criterios pre-establecidos intenta identificar con mayor seguridad una bacteriemia genuina ${ }^{11}$, punto importante en un estudio de esta naturaleza que busca explicar de mejor forma la etiología de los episodios.

Escherichia coli fue el BGN más frecuentemente identificado como productor de bacteriemia en $\mathrm{NF}^{18}$, seguido de Klebsiella sp y luego Pseudomonas sp. De acuerdo a datos publicados en la literatura médica, la frecuencia de bacteriemia por Pseudomonas sp es variable, en algunos centros se reporta hasta en $18 \%$ de los episodios de $\mathrm{NF}^{21,22}$, en tanto en otros se describen frecuencias cercanas al $3 \%^{3,14,18,23}$. No se reconoce una clara explicación para estas diferencias o si existe una tendencia a disminuir la frecuencia de su identificación, algunos autores han planteado que podría existir una prevalencia cíclica ${ }^{18}$. Este punto reviste particular interés ya que habitualmente la antibioterapia empírica inicial considera cobertura anti-pseudomonas. $\mathrm{Si}$ bien en nuestro centro representa el 8,2\% de las bacteriemias demostradas, debemos ser cautos en la interpretación de estos datos; es prudente analizar un 
período de vigilancia mayor, que permita reconocer tendencias y factores de riesgo antes de recomendar cambios en la cobertura de los esquemas antimicrobianos empíricos. La frecuencia de 22,2\% de BGN resistentes a cefalosporinas de $3^{\mathrm{a}}$ generación duplica la frecuencia descrita en bacteriemias por BGN de pacientes hospitalizados en nuestro centro ${ }^{24}$, lo cual podría correlacionarse a hospitalizaciones previas o uso de antimicrobianos en forma reciente ${ }^{25}$. Deben explorarse en forma dirigida los factores de riesgo de resistencia bacteriana en pacientes oncológicos, nuestros datos sugieren que el grupo que presenta identificación de BGN BLEE positivos tiene más frecuentemente hospitalizaciones previas, pero es un dato insuficiente ya que desconocemos la duración de las estadías previas, NF anteriores o uso previo de antimicrobianos. Un factor que podría influir en la resistencia bacteriana es el uso de antibioprofilaxis, parece importante vigilar los cambios epidemiológicos en un nuevo escenario como es el uso, cada vez más frecuente, de profilaxis antimicrobiana con levofloxacina, aplicado en diferentes centros ${ }^{26}$. Por otra parte, la mayor frecuencia de BGN resistentes nos hace plantear que la monoterapia con cefalosporinas sería inadecuada en una proporción de nuestros pacientes, siendo preferible la asociación con aminoglucósidos hasta la obtención de los hemocultivos con identificación bacteriana y estudio de susceptibilidad in vitro.

La detección de cuatro candidemias en esta serie nos confirma que se trata de un hecho infrecuente ${ }^{4}$; no obstante, sería útil establecer quiénes deben recibir terapia antifúngica empírica desde el inicio del episodio de NF. Existen algunos factores de riesgo "clásicos" para adquirir candidiasis invasora que se podrían considerar en la evaluación de estos pacientes ${ }^{27}$.

Se debe destacar el hallazgo de virus respiratorios en 13 episodios; cabe la posibilidad de sub-diagnóstico de mayores casos por falta de sospecha clínica oportuna. No se han publicado otras experiencias nacionales al respecto en pacientes adultos. Un estudio prospectivo en niños chilenos demostró infección por virus respiratorios: influenza, parainfluenza, respiratorio sincicial o adenovirus en $25 \%$ de los episodios de $\mathrm{NF}^{28}$, lo que plantea la necesidad de buscar más frecuente y dirigidamente la infección por virus respiratorios en estos pacientes.

La mayor mortalidad observada en los pacientes en que se logra documentar microbiológicamente una infección plantea la necesidad de mayores estudios para definir factores de riesgo de esta condición en nuestro medio. En pediatría se ha logrado identificar factores predictores de infección bacteriana invasora ${ }^{29}$ y en adultos escalas de predicción de riesgo de complica- ciones $^{30}$ además de factores de riesgo de infección por CGP, BGN y hongos ${ }^{19,27,31}$; su aplicabilidad en nuestro medio o el diseño de nuevas escalas con diferentes factores de riesgo debe ser evaluada en futuros estudios.

Respecto a los episodios catalogados como FOD, se debe comentar que existen otras causas de fiebre en neutropenia incluyendo causas no infecciosas, pero también se debe aceptar las limitaciones en el estudio microbiológico, pueden quedar no diagnosticadas infecciones virales y bacteriemias por anaerobios. Es un campo a explorar las metodologías de laboratorio microbiológico a emplear en estos pacientes de modo de incrementar nuestro diagnóstico. Esto es de suma importancia ya que si logramos estar seguros de que no existe infección bacteriana en algunas circunstancias podríamos simplificar o suspender la terapia antimicrobiana.

La demostración de mayor gravedad de los episodios de NF en $\mathrm{CH}$ respecto a COS, mayor frecuencia de bacteriemia, complicaciones y mortalidad como se describe en otros estudios ${ }^{16,30}$, plantea que el enfrentamiento en este grupo debe ser más enérgico, en especial en la prontitud de inicio de antibioterapia apropiada.

De nuestros datos no podemos inferir cuál sería la utilidad de implementar profilaxis antimicrobiana con levofloxacina en pacientes neutropénicos en nuestro centro. Si bien hay evidencia que apoyan su uso, especialmente en los primeros ciclos de quimioterapia $^{32}$, su eficacia ha sido cuestionada ${ }^{33,34}$; se requeriría un estudio especialmente diseñado para ver los reales beneficios de esta práctica en nuestro medio y objetivar el real impacto sobre la emergencia de resistencia bacteriana.

Este estudio tiene limitaciones, el estudio microbiológico y radiológico no se ciñen a un protocolo, no están los datos de exposición previa a antimicrobianos ni se consideró la estratificación de riesgo. Si bien este estudio se refiere sólo a la experiencia de un centro, demuestra la importancia de la vigilancia de los episodios de NF para definir conductas locales; la generalización de estos datos no es posible, pero pueden ser orientadores para centros que atiendan pacientes adultos con patologías similares.

En conclusión, queda establecido el perfil etiológico de la NF en nuestro centro: alta frecuencia de documentación de infección, predominio etiológico de BGN sobre CGP y mayor frecuencia a lo esperado de identificación de BGN resistentes a cefalosporinas de $3^{\mathrm{a}}$ generación. Esto tiene implicancias en la elección de terapia antimicrobiana empírica inicial, plantea la necesidad de nuevos estudios que permitan identificar factores de riesgo de infecciones invasores que tendrían 
peor pronóstico y la necesidad de mantener una vigilancia activa de los episodios de NF.

\section{Resumen}

Introducción: La vigilancia de la etiología de los episodios de neutropenia febril (NF) en cada centro permite adaptar guías de antibioterapia a la epidemiología local. Objetivo: Caracterizar y comparar la etiología de la NF en pacientes con cáncer hematológico $(\mathrm{CH})$ y de órganos sólidos (COS). Pacientes y Métodos: Estudio de vigilancia de NF de pacientes adultos en el Hospital Clínico Universidad Católica, en Santiago, Chile, entre enero 2004 y agosto 2007. Resultados: 154 episodios de NF correspondientes a 87 pacientes: $47 \pm 6$ años; $71 \% \mathrm{CH}$ y $29 \%$ COS. Se documentó infección clínica y/o microbiológicamente en $76 \%$. Más frecuente fueron: foco gastrointestinal $31,5 \%$, respiratorio alto $30,3 \%$ y respiratorio bajo 16,9\%. En $30,5 \%$ hubo hemocultivos positivos: bacilos gramnegativos en $51 \%$, cocáceas grampositivas en $41 \%$, levaduras en $8 \%$; predominando: Escherichia coli 22\%, Staphylococcus coagulasa negativa (SCoN) 20\% y Klebsiella pneumoniae $12 \% ; 22,2 \%$ de las enterobacterias eran productoras de $\beta$-lactamasa de espectro expandido y $55,6 \%$ SCoN meticilina resistentes. En $18,3 \%$ de los episodios no se identificó causa de fiebre. Hubo mayor mortalidad en episodios con documentación microbiológica $(14,5 \%$ vs $1,3 \%, \mathrm{p}<0,005)$. En los pacientes con $\mathrm{CH}$ fue más frecuente obtener hemocultivos positivos $(37,3 \%$ vs $13,6 \%$; p < 0,01) e identificar foco clínico $(67,2 \%$ vs $50 \%$; $\mathrm{p}=0,045)$. Conclusiones: Se establece el perfil etiológico de las NF en nuestro centro y la necesidad de mantener vigilancia. En futuros estudios será necesario evaluar factores de riesgo de pacientes con infecciones invasores que tendrían peor pronóstico.

\section{Referencias}

1.- Dale D C. Colony-stimulating factors for the management of neutropenia in cancer patients. Drugs 2002; 62: S1-15.

2.- Pizzo P A, Robichaud K J, Wesley R, Commers J R. Fever in the pediatric and young adult patient with cancer: A prospective study of 1001 episodes. Medicine 1982; 61: 153-65.

3.- Wisplinghoff H, Seifert H, Wenzel R P, Edmond M B. Current trends in the epidemiology of nosocomial bloodstream infections in patients with hematological malignancies and solid neoplasms in hospitals in the United States. Clin Infect Dis 2003; 36: 1103-10.

4.- Hughes W T, Armstrong D, Bodey G P, Bow E J, Brown A E, Calandra T, et al. Guidelines for the use of antimicrobial agents in neutropenic patients with cancer. Clin Infect Dis 2002; 34: 730-51.

5.- Marchetti $O$, Calandra $T$. Infections in neutropenic cancer patients. Lancet 2002; 359: 723-5.

6.- Bochud P Y, Calandra T, Francioli P. Bacteremia due to viridans streptococci in neutropenic patients: a review. Am J Med 1994; 97: 256-64.

7.- Yadegarynia D, Tarrand J, Raad I, Rolston K. Current spectrum of bacterial infections in patients with cancer. Clin Infec Dis 2003; 37: 1144-5.

8.- Puga B, Puga I, Cabrera M E, Undurraga M S, Guerra C, Urrejola G, et al. Neutropenia febril de alto riesgo en leucemia aguda.
Experiencia en un hospital público: Programa Nacional de Drogas Antineoplásicas del Adulto (PANDA), Hospital del Salvador, 1991-2001. Rev Méd Chile 2003, 131: 1023-30.

9.- Santolaya M E, Rabagliati R, Bidart T, Paya E, Guzmán A M, Morales R, et al. Consenso: Manejo racional del paciente con cáncer, neutropenia y fiebre. Rev Chil Infect 2005; 22: S79-113.

10.- WHO handbook for reporting the results of cancer treatment. WHO Offset Publications, Geneva 1979; Series number 48.

11.- García P, Benítez R, Lam M, Salinas A M, Wirth H, Espinoza C, et al. Coagulasenegative staphylococci: clinical, microbiological and molecular features to predict true bacteraemia. J Med Microbiol 2004; 53: 67-72.

12.- Ramos G, Domínguez I, Benítez R, Guzmán A M, García P, Sánchez T, et al. Etiología de bacteriemias en neutropenia febril de pacientes adultos con cáncer. Hospital Clínico Universidad Católica 20012004. XXI Congreso Chileno de Infectología. Viña del Mar 13-16 de octubre 2004. Libro de Resúmenes. Pág 42, resumen CO1.

13.- Vencer S, Salepci T, Ozer A. Evaluation of infectious etiology and prognostic risk factors of febrile episodes in neutropenic cancer patients. J Infect 2003; 47: 65-72.

14.- Toussaint E, Bahel-Ball E, Vekemans M, Georgala A, Al-Hakak L, Paesmans M, et al. Causes of fever in cancer patients (prospective study over 477 episodes)
Support Care Cancer 2006 14: 763-9.

15.- Klastersky J, Ameye L, Maertens J, Georgala A, Muanza F, Aoun M, et al. Bacteraemia in febrile neutropenic cancer patients. Int J Antimicrob Agents 2007; 30: S51-9.

16.- Bodey G P. Unusual presentations of infection in neutropenic patients. Intl $\mathrm{J}$ Antimicrob Agents 2000; 16: 93-5.

17.- Klastersky J. Science and pragmatism in the treatment and prevention of neutropenic infection. J Antimicrob Chemother 1998; 41: $13-24$

18.- Ramphal R. Changes in the etiology of bacteremia in febrile neutropenic patients and the susceptibilities of the currently isolated pathogens. Clin Infect Dis 2004; 39: S25-31.

19.- Cordonnier C, Buzyn A, Leverger G, Herbrecht R, Hunault M, Leclercq R, et al Epidemiology and risk factors for grampositive coccal infections in neutropenia: toward a more targeted antibiotic strategy. Clin Infect Dis 2003; 36: 149-58.

20.- Engelhard D, Elishoov H, Strauss N, Naparstek E, Nagler A, Simhon A, et al Nosocomial coagulase-negative staphylococcal infections in bone marrow transplantation recipients with central vein catheter: A 5-year prospective study. Transplantation 1996; 61: 430-4.

21.- Maschmeyer G, Braverny I. Review of the incidence and prognosis of Pseudomonas aeruginosa infections in cancer patients in the 1990s. Eur J Clin Microbiol Infect Dis 2000; 19: 915-25. 
22.- Rolston K V, Tarrand J J. Pseudomonas aeruginosa-still a frequent pathogen in patients with cancer: 11-year experience at a comprehensive cancer center. Clin Infect Dis 1999; 29: 463-4.

23.- Rolston K V. Challenges in the treatment of infections caused by gram-positive and gram-negative bacteria in patients with cancer and neutropenia. Clin Infect Dis 2005; 40: S246-52.

24.- García P, Rubilar C, Vicentini D, Labarca J. Caracterización clínica de las bacteriemias por enterobacterias productoras de $\beta$-lactamasas de espectro extendido en un hospital universitario. XXIV Congreso Chileno de Infectología. Pucón 14-17 de noviembre 2007. Libro de Resúmenes. Pág 70, resumen P16.

25.- Sipsas N, Bodey G P, Kontoyiannis D P. Perspectives for the management of febrile neutropenic patients with cancer in the 21 st century. Cancer 2005; 103: 1103-13.

26.- Cattaneo C, Quaresmini G, Casari S, Capucci M A, Micheletti M, Borlenghi E, et al. Recent changes in bacterial epidemiology and the emergence of fluoroquinolones-resistant Escherichia coli among patient with haematological malignancies: result of a prospective study in 823 patients in a single institution. J Antimicrob Chemother 2008; 61: 721-8.

27.- Anaissie E J, Rex J H, Usun O, Vartivarian S. Predictors of adverse outcome in cancer patients with candidemia Am J Med 1998; 104: 238-45.

28.- Täger M, Zolezzi P, Folatre I, Navarrete M, Rojas J. Infecciones por virus respiratorios en niños con leucemia linfoblástica aguda y neutropenia febril: Estudio prospectivo. Rev Chil Infect 2006; 23: 118-23.

29.- Santolaya M E, Alvarez A M, Avilés C L, Becker A, Cofré J, Enríquez N, et al. Prospective evaluation of a model of prediction of invasive bacterial infection risk among children with cancer, fever, and neutropenia. Clin Infect Dis 2002; 35: 678-83.

30.- Klastersky J, Paesmans M, Rubenstein E B,
Boyer M, Elting L, Feld R, et al. The multinational association for supportive care in cancer risk index: A multinational scoring system for identifying low-risk febrile neutropenic cancer patients. J Clin Oncol 2000; 18: 3038-51.

31.- Cordonier C, Herbrecht R, Buzyn A, Leverger G, Leclercq R, Nitenberg $G$ et al. Risk factors for gram-negative bacterial infection in febrile neutropenia. Hematologia 2005, 90; 1102-9.

32.- Cullen M, Steven N, Billingham L, Gaunt C, Hasting M, Simmonds P et al. Antibacterial prophylaxis after chemotherapy for solid tumors and lymphomas. N Engl J Med 2005; 353: 988-98

33.- Freifeld A G, Sepkowitz K A, Almyroudis N G, Segal B H, Castagna L, Santoro A et al. Antibacterial prophylaxis in patients with cancer and neutropenia. N Engl J Med 2006; 354: $90-2$.

34.- Rabagliati R, Bidart T, Santolaya M E. Uso de profilaxis antibiótica en pacientes neutropénicos. Rev Méd Chile 2006; 134: $801-2$. 